\title{
Analyse logico-rhétorique du discours de Guillaume Du Vair à Marie de Médicis, nouvelle reine de France
}

Notes, édition du texte et traduction de Giuliano Ferretti et Francis Goyet

\section{Abraham Vechner}

Giuliano Ferretti et Francis Goyet (éd.)

Traducteur : Giuliano Ferretti et Francis Goyet

\section{(2) OpenEdition} Journals

\section{Édition électronique}

URL : http://journals.openedition.org/rhetorique/336

DOI : 10.4000/rhetorique.336

ISSN : 2270-6909

\section{Éditeur}

UGA Éditions/Université Grenoble Alpes

\section{Édition imprimée}

ISBN : 978-2-84310-274-5

Référence électronique

Abraham Vechner, «Analyse logico-rhétorique du discours de Guillaume Du Vair à Marie de Médicis, nouvelle reine de France », Exercices de rhétorique [En ligne], 3 | 2014, mis en ligne le 16 juin 2014 , consulté le 12 septembre 2020. URL : http://journals.openedition.org/rhetorique/336 ; DOI : https:// doi.org/10.4000/rhetorique.336

Ce document a été généré automatiquement le 12 septembre 2020.

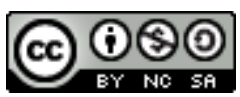

Les contenus de la revue Exercices de rhétorique sont mis à disposition selon les termes de la Licence Creative Commons Attribution - Pas d'Utilisation Commerciale - Partage dans les Mêmes Conditions 4.0 International. 


\section{Analyse logico-rhétorique du discours de Guillaume Du Vair à Marie de Médicis, nouvelle reine de France}

Notes, édition du texte et traduction de Giuliano Ferretti et Francis Goyet

\section{Abraham Vechner}

Giuliano Ferretti et Francis Goyet (éd.)

Traduction : Giuliano Ferretti et Francis Goyet

Auteur : Abraham Vechner (1636-1704).

Texte des p. 446-449 de l'unique édition, Suada Gallicana, h. e. conciones et orationes Thuanaeae [...]. Accedit Analyseos Logico-Rhetorico Specimen duplex [...], Francfort et Leipzig, J. Adam Kästner, 1679. Soit : L'Éloquence française, c'est-à-dire harangues et discours de de Thou [...]. Avec un double spécimen d'analyse logico-rhétorique. Numérisation intégrale sur http://digital.staatsbibliothek-berlin.de/dms/ werkansicht/?PPN=59285261X\&PHYSID=PHYS_0483 (accès direct)

Ces pages 446-449 sont la seconde partie du « double Spécimen » annoncé au titre. Vechner y propose l'analyse détaillée d'un discours de 1600, par lequel Guillaume Du Vair souhaite la bienvenue à Marie de Médicis, qui vient de débarquer à Marseille pour épouser Henri IV.

Notre présentation est la suivante : d'abord le texte même du discours analysé ; puis l'analyse par Vechner.

Le discours a été rédigé en latin par de Thou (1553-1617). Nous reprenons la première traduction disponible de ce texte latin, laquelle date de 1734 . Nous aérons cette traduction française en la divisant en paragraphes, lesquels correspondent aux quatre parties de l'analyse : exorde, arguments, thèse, 
conclusion. De même, nous y insérons, entre crochets droits, les mêmes numéros et repères que ceux de l'analyse. On trouvera plus haut dans le « Dossier » (annexe de l'article de Giuliano Ferretti) la version française du même discours, publiée en plaquette dès 1600 par Du Vair lui-même, et dont de Thou s'est très certainement inspiré.

Pour l'analyse même de Vechner, nous suivons les mêmes principes d'édition que pour le discours précédent. Les mots grecs donnés par Vechner en caractères grecs sont ici syncharistique, hypophore et icône.

\section{Discours de Du Vair (1600), chez de Thou'}

1 Le Chancelier porta la parole en présence du Connétable, et complimenta la Reine au nom du Roi sur son heureuse arrivée en France. Le même jour du Vair premier Président du Parlement de Provence, conduit par le Chancelier, eut audience de S. M. qu'il complimenta au nom de sa Compagnie. On dit qu'il tint ce discours.

\section{[Exorde]}

« [ $\alpha]$ Madame, au bruit de l'heureuse arrivée de V. M. très Chrétienne dans ce Royaume, dont elle vient faire le bonheur, nous quittons tous le temple de Thémis et le sanctuaire de la Justice, [ $\beta]$ pour venir nous prosterner à vos pieds, et pour donner à tous vos sujets l'exemple de la soumission et des hommages, qui sont dus à V. M. Établis pour les contenir dans l'ordre, il est bien juste que nous soyons les premiers à accomplir, en nous acquittant du plus glorieux et du plus honorable de tous les devoirs, les vœux que nous avons faits au Seigneur pour vous posséder.

[ $\alpha$ ] Ad optatissimi Galliae toti adventus famam, Christianissima Regina, ecce Themidis adyta, justitiae sacraria \& penetralia cuncti deserimus, [ $\beta]$ ut pedibus Tuis advoluti, qui alios ad obsequium cogimus, obedientiae Tuae Majestati praestandae exemplo praeiremus, \& fidem voti relligione obligatam hoc longe honorificentissimo officio defuncti exsolveremus.

\section{[Arguments]}

3 [1] Oui, Madame, V. M. remplit tous les désirs de la France, votre auguste présence ne nous laisse plus rien à souhaiter; et pour rendre notre bonheur parfait, il ne reste plus, sinon que la bonté divine nous conserve ses présents, et nous fasse jouir longtemps de la félicité qu'il nous procure, par l'arrivée de votre personne sacrée. \$ [2] Le Ciel nous a donné un Roi excellent en vertu, admirable en bonté, incomparable en valeur, qui a assuré le repos de la France, par les travaux qu'il a essuyés ; la tranquillité de ses sujets, par les dangers auxquels il s'est exposé ; et la gloire de la Nation, par les victoires qu'il a remportées. Nous eussions été le peuple le plus heureux de l'Univers, si une réflexion fâcheuse n'eût point troublé notre joie et notre bonheur, en nous faisant souvenir que la nature a borné le cours de la vie humaine ; qu'il n'y a rien de durable ici-bas; et que le temps nous enlèverait quelque jour un Prince, à qui il n'y avait aucun de nous, qui n'eût souhaité l'immortalité. Nous ne pouvions penser, sans une tristesse accablante, que cette vie solitaire et privée des douceurs qui accompagnent le mariage, à laquelle ce Prince semblait condamné, lui rendait à lui-même ennuyeux et désagréables des 
jours, qui sont si précieux à tous ses sujets, et lui ôtait l'espérance de laisser un héritier, à qui la France eût un jour autant d'obligation, qu'elle en avait déjà au père. § Nous allions succomber au déplaisir que nous causait la vue d'un avenir si fâcheux, lorsque le Ciel vous a envoyée à notre secours. Oui, Madame, l'éclat de V. M. a dissipé les nuages de la tristesse qui nous accablait. Le repos et la sûreté dont nous jouissons sous un si bon Prince, nous remplissait de joie ; mais notre joie n'était point parfaite. Nous ne la possédions que comme un dépôt. Vous venez de l'assurer. Vous la rendez durable par l'espérance dont nous osons nous flatter, qu'avec la grâce du Seigneur, vous rendrez à la famille Royale l'ordre d'une succession légitime, qui a été interrompue depuis si longtemps; et qu'il sortira de vous, pour la sûreté d'un trône ébranlé par tant de secousses, une postérité nombreuse digne d'un Royaume si puissant, et en qui nous verrons revivre la valeur du père, et les vertus admirables de la mère.

[1] In Te Tuoque adventu desideria omnia publica, cuncta vota exhausta, \& consumpta sunt, nihilque ad summae felicitatis cumulum deest, nisi, ut quae DEUS O. M. bona contulit, propria deinceps faciat, \& felicitate, per Te concessa, quam diutissime fruamur. [2] Rex nobis contigit, virtute praestans, benignitate admirabilis, fortitudine incomparabilis, qui laboribus suis Regno quietem, periculo proprio securitatem, victoriis gloriam conciliavit. Verum in tanta seu laetitia, sive felicitate non nunquam tristis cogitatio subibat, humanae conditionis fineis a natura praestitutos esse, nihilque sub coelo perpetuo durare, \& quod cuncti assiduis votis immortale exoptabant, aliquando desiturum. Solitudinem vero illam coelibatum, in quo Rex degebat, vitam ipsi, quae cunctis adeo cara erat, minus gratam reddere, \& spem haeredis, cui, quantum Patri debebat, tantum olim debitura esset Gallia, adimere. In hac futuri anxia cogitatione defixis quasi coelo demissa nobis illuxisti, cunctasque tristitiae nubeculas vultus Tui splendore discussisti, \& tranquillitatis, qua sub optimo Principe quasi precario fruebamur, solidum gaudium fecisti, spe haud dubia fore, ut, adspirante Dei gratia, in familia Regia intermortuae per tot annos successionis spes per te renovetur, \& digna tanto regno numerosa progenies, quae Patrem virtute, Matrem sanctissimis moribus referat, ad regni alioqui nutantis fulcimentum succrescat.

\section{[Thèse]}

4 Ainsi, pleins du plus profond dévouement et de la plus respectueuse reconnaissance, nous célébrons avec joie votre heureuse arrivée en France. Fasse le Ciel, que vous régniez longtemps, et pour le bonheur d'une Nation qui vous reconnaît aujourd'hui pour sa Reine! Que la fin du siècle où nous entrons, puisse vous voir heureuse épouse d'un Roi heureux! Que la postérité la plus reculée admire en vous l'heureuse mère de tant de Rois, à qui vous donnerez la naissance.

Proinde devoti Numini Majestatique Tuae faustum felicemque tuum ad littus Gallicum appulsum gratulamur, DEUMque supplices comprecamur, ut diu apud nos \& nobis feliciter regnes: ut seculum, quod nunc ingredimur, cum desinet, Te cum Rege marito superstite incolumem videat, \& quae ventura sunt, beatam tot Regum ex Te nasciturorum matrem praedicent.

\section{[Arguments, suite et fin]}

$5 \$$ [3] Vous venez, Madame, pour être l'épouse d'un grand Roi, qui n'a presque point eu d'égal dans tous les siècles passés. Vous apportez dans sa maison la grandeur de la maison d'Autriche dont vous sortez, la sagesse et la prudence de la maison de Médicis dont vous portez le nom. 
[3] Magni Regis, et cui vix parem priora secula habuerunt, Uxor venis, magnitudinem Austriae ac familiae, unde orta es, prudentiam, sapientiam Medicaeae, unde nomen trahis, Tecum in domum ejus inferens.

\title{
[Conclusion]
}

6 Souvenez-vous aussi, et n'oubliez jamais, que Dieu vous appelle par cette alliance, à devenir non seulement la Reine la plus puissante qui soit dans le monde Chrétien, mais encore la mère la plus tendre et la plus compatissante de tant de peuples, dont le Roi votre époux est le père. Ainsi, comme vous allez partager avec lui sa Couronne, songez de même à prendre part aux soins glorieux que le trône exige de lui. Aimez des peuples sur lesquels vous allez régner avec lui. Aimez des sujets dont la conservation et la sûreté occupent sans cesse toutes ses pensées; et aimez-les avec la même tendresse qu'il a pour la nation Française, à laquelle vous venez d'être unie sous des présages si heureux ; afin que non seulement nous vous obéissions avec joie ; mais que nos voisins mêmes se croient redevables de leur bonheur aux exemples de V.M. lorsqu'ils se verront gouvernés par leurs Souverains, avec la même bonté et la même affection. »

\begin{abstract}
Vicissim memor esto, adque Tibi multoties repetitum puta, non solum Te ad potentissimae toto Christiano orbe Reginae titulos, sed etiam ad beneficentissimae tot populorum, quorum Rex Pater est, Matris munus per hoc matrimonium a Deo vocatam. Age ergo, \& ut tori consors, sic regiae sollicitudinis in posterum particeps hanc gloriam occupa, \& populos imperio communi subditos, de quorum salute \& quiete assidue Rex cogitat, pari erga FrancoGallicum nomen, cui nunc omine felici inserta es, caritate prosequere : ut laeti non solum ipsi Tibi serviant, sed vicini, eandem felicitatem cum a suis Principibus adepti fuerint, eam se Tuo exemplo debere fateantur.
\end{abstract}

7 Après ce discours du premier Président, la Reine pria Dieu de lui faire la grâce de répondre aux désirs et aux espérances de la Nation, et remercia tous ceux qui étaient présents.

\section{Analyse logico-rhétorique², par Vechner}

8 Ce Discours relève du genre Démonstratif, et plus spécialement de cette sous-catégorie qu'est < d'un mot grec > un discours syncharistique ${ }^{3}$, ou si l'on préfère <en latin> un discours de congratulation ${ }^{4}$ : en tant que premier président du Parlement de Marseille, sur un ton de vénération très humble, l'orateur congratule la Reine de France MARIE DE MÉDICIS pour son heureuse arrivée en France.

Generis Caussarum Demontrativi haec est Oratio, atque in specie quidem sugkharistikè ${ }^{5}$, sive gratulatoria: in ea siquidem provinciae Massiliensis Praeses primarius MARIAE MEDICAEAE, Galliarum Reginae, felicem in Galliam ingressum humillima animi veneratione gratulatur.

Le discours se compose de quatre parties.

Quaternione vero partium absolvitur : quarum

\section{[Exorde]}

10 La première partie est l'Exorde, qui tire argument de l'accident ou de l'effet : pourquoi les Sénateurs ${ }^{6}$ de Marseille ont-ils déserté en masse leurs Tribunaux? pour plusieurs causes qui sont indiquées : 
Primam constituit Exordium, ab accidente sive effectu Senatorum Massiliensium, Dicasteriorum nimirum desertione, ductum : cujus rei notantur caussae

a)

11 la cause directe qui les y a poussés, à savoir le bruit de l'arrivée d'une nouvelle Reine ;

a) impulsiva, adventus Reginae novae fama ; [p. 447]

及)

12 une cause finale double ${ }^{7}$ : en direction du public, montrer l'exemple de l'obéissance qu'on doit à une Reine; en direction de la reine elle-même, lui déclarer hautement la foi qui lui est due, ou si l'on préfère s'acquitter de ce devoir; ce à quoi il assigne une cause instrumentale, l'honneur extrême d'avoir été chargés de la recevoirs. Les mots correspondant <à ces $1^{\mathrm{er}}$ et $2^{\mathrm{e}}>$ sont « Madame, au bruit de l'heureuse arrivée... », etc.

3 ) finalis gemina, partim exempli obedientiae Reginae dictae praestandae editio ; partim fidei debitae declaratio, sive exsolutio: cujus caussa intrumentalis adjicitur, istud puta honorificentissimum exceptionis officium. Quo spectant verba: Ad optatissimi Galliae toti adventus etc.

\section{[Thèse]}

13 La deuxième partie est la Thèse, qui contient la congratulation pour l'heureuse arrivée de la Reine sur le rivage français. En même temps qu'il pose cette thèse, il exprime son argument ou si l'on préfère le sommaire ${ }^{9}$ de son argument, par l'effet <que produit cette heureuse arrivée>, à savoir par le vœu d'un long et heureux règne. Il lui assigne une double cause finale. La première est de voir la Reine auprès du Roi son époux pendant toute la durée de ce siècle, en vie et en bonne santé l'un et l'autre; la seconde est l'admiration de voir la Reine être l'heureuse Mère de tant de Rois; et la cause efficiente $<$ de cette admiration ou célébration>, ce sont les siècles à venir ${ }^{10}$. Voir les mots : « Ainsi, pleins du plus profond dévouement... » jusqu'à « la naissance ».

Secundam Propositio, gratulationem Reginae ob felicem ad litus Gallicum adpulsum factam continens : cujus insimul argumentum sive summa exprimitur, petita ab effectu, diuturni videlicet ac felicis in Gallia regiminis voto: cujus ipsius duplex caussa finalis additur, una scilicet Reginae cum Rege marito per seculum istud integrum incolumitas et vita; altera Reginae, uti beatae tot Regum ex ipsa nasciturorum Matris, praedicatio : cujus quidem rei caussa efficiens secula ventura. Vide verba: Proinde devoti Numini Majestatique Tuae etc. usque ad verbum praedicent.

\section{[Arguments]}

La troisième partie est l'Argumentation:

Tertiam partem constituit Confirmatio, petita

1)

par la cause qui pousse directement à cette joie générale, ou si l'on préfère par un utile double. Le premier est le fait que tous les désirs et les vœux publics soient comblés par l'arrivée d'une nouvelle reine; le second est le sommet ou la grandeur d'une félicité dont du Vair exprime le souhait qu'elle soit pour fort longtemps le bien du royaume ${ }^{11}$. Les mots <correspondant à ce $1^{\mathrm{e}}>$ sont : « Oui, Madame, Votre Majesté... » et suivants. 
1) a caussa impulsiva, sive utili gemino, priore quidem desideriorum ac votorum publicorum in Reginae novae adventu facta adimpletione; posteriore felicitatis, quam propriam regno et diuturnam precatur Varius, apice, seu magnitudine, verbis subsequentibus: In Te Tuoque adventu, et reliquis. [p. 448]

2)

par une circonstance ou par un effet de son arrivée, ou encore par l'agréable, à savoir ce bonheur solide dont ils ont l'assurance de pouvoir désormais jouir tranquillement. Il lui assigne comme cause efficiente la naissance à venir de nombreux enfants qui garantissent une succession légitime au Roi, ce qui est leur espoir à tous : naissance dont il indique implicitement ${ }^{12}$ ce qui en est la cause efficiente principale, à savoir la grâce divine. Cet argument est illustré avec éclat par le lieu du contraste des opposés, sous la forme d'une Réponse qui prévient par avance une objection ${ }^{13}$. Le contraste se fait < d'un mot grec> par une hypophore <ou objection implicite ${ }^{14}>$ au bonheur que représentent les vertus du roi, ses hauts faits militaires et ses bienfaits répandus sur le royaume : c'està-dire par la peur et la tristesse à l'idée que puisse cesser un jour ce bonheur actuel, alors que tous priaient pour qu'il soit immortel. Il assigne une double cause qui pousse directement <à cette peur et tristesse>. La première est une réflexion sur la condition humaine : la nature a borné le cours de notre vie par des limites fixées d'avance. La seconde cause est le célibat du Roi, qui fait de lui un solitaire : avec pour effet aussitôt ajouté comme un corollaire, l'absence d'espoir d'un héritier qui puisse lui succéder. Les mots <correspondant à ce $2^{\mathrm{e}}>$ vont de « Le Ciel nous a donné un Roi... » jusqu'à « de la mère ".

2) ab adjuncto seu effectu adventus ejus, vel jucundo, solidi nimirum gaudii fruendae tranquillitatis excitatione: cujus caussa efficiens adjungitur, spes videlicet omnium haud dubia prolis numerosae, atque legitimi adeo successoris Regi ex Regina nascituri : cujus rei caussa efficiens principalis connotatur, divina nempe gratia. Illustratur autem hoc argumentum ex loco ab opposito per Occupationem, sumpto a Regis virtutibus et rebus gestis ac meritis in regnum hupophorâa ${ }^{15}$, metu scilicet et tristitia, ne, quod immortale precabantur omnes, desineret aliquando : cujus rei duplex adjicitur caussa impellens, prior quidem, humanae conditionis meditatio : cui certi a natura praestituti sunt fines; posterior, Regis caelibatus ac solitudo : cujus effectus subjungitur, spei scilicet haeredis ac successoris ablatio, a verbis : Rex nobis contigit etc. usque ad verbum succrescat.

3)

par les circonstances de la Reine elle-même, ou si l'on préfère par l'honorable et le glorieux :

3) ab adjunctis ipsius Reginae, sive honesto ac glorioso, quod nimirum ipsa

a) la grandeur du Roi dont elle vient pour être l'Épouse, une grandeur presque sans égale ;

a) magni et incomparabilis fere Regis Uxor veniat ;

19 ß) la grandeur de la famille d'Autriche dont elle est issue, ainsi que la prudence et sagesse <politiques> des Médicis dont elle porte le nom, <grandeur et sagesse> qui vont grâce à elle enrichir le lignage du Roi.

ß) magnitudinem familiae Austriacae, ex qua orta, uti et prudentiam ac sapientiam Medicaeae, unde ipsi nomen, in domum Regis inferat.

20 Les mots correspondant <à ce $3^{\mathrm{e}}>$ sont « Vous venez, Madame, pour être l'épouse... » et ce qui suit. 
Quo referenda sunt: Magni Regis, et quae sequuntur.

\section{[Conclusion]}

La quatrième partie enfin est la Conclusion, tirée de l'effet d'un Dirigeant <à savoir, aimer ses sujets ${ }^{16}>$. Il exhorte ${ }^{17}$ donc la Reine à aimer ses sujets, en prenant modèle sur le Roi son époux. Il ajoute la double finalité de cet amour, ou si l'on préfère l'utile et le glorieux. La première finalité est d'obtenir l'obéissance des sujets, mais une obéissance dans la joie (grâce aux <douces> méthodes de la Reine ${ }^{18}$ ). La seconde finalité est de viser la gloire, celle de voir les peuples voisins proclamer qu'ils doivent leur félicité à l'exemple qu'elle aura donné à leurs propres souverains. L'argument de cette exhortation est tiré de la cause directe qui pousse à ainsi aimer ses sujets, à savoir sa vocation divine <car elle est appelée par Dieu ${ }^{19}>$ non seulement aux titres de Reine la plus puissante de tout le Monde Chrétien, mais aussi à la charge <écrasante> d'être la Mère de tant de peuples, à l'image du Roi qui est un vrai Père pour eux. Ce dont la cause organique ${ }^{20}$ ou le moyen est ce mariage lui-même. Les mots correspondants sont "Souvenez-vous aussi et n'oubliez jamais... » jusqu'à la fin du Discours ${ }^{21}$.

[p. 449] Quartam denique constituit Conclusio, deprompta ab effectu Praesidis, Reginae puta ad amorem subditorum exemplo Regis cohortatione: cujus quidem amoris finis geminus, sive utile ac gloriosum subjungitur, prior nempe laeta subditorum (ratione Reginae) servitia; posterior, vicinorum populorum, suam se ipsius exemplo felicitatem debere, professio. Argumentum vero istius cohortationis ducitur a caussa impulsiva, divina nimirum non ad titulos modo potentissimae toto Christiano Orbe Reginae; sed ad munus etiam Matris tot populorum, Regi, tanquam Patri, subditorum, vocatione: cujus caussa organica seu medium est illud ipsum matrimonium. Conferantur verba textus: Vicissim memor esto, usque ad calcem Orationis.

\section{Ornements :}

Ornatum conciliant

dans l'Exorde, une Périphrase, une Métonymie de la cause externe efficiente pour l'effet ${ }^{22}$ et une Antithèse ;

Exordio quidem Periphrasis, Metonymia caussae externae efficientis pro effectu, et Antitheton;

dans la Thèse, des Métaphores, une Métonymie, un Polyptote, etc. ;

Propositioni Metaphorae, Metonymia, Polyptoton etc.

dans l'Argumentation, plusieurs Métaphores, une Prolepse, un Homéotéleute, des Antithèses, une Icône ou si l'on préfère <le mot latin> une Image, et une Allégorie ${ }^{23}$;

Confirmationi Metaphorae complures, Prolepsis, Homoeoteleuton, Antitheta, Eikôn ${ }^{24}$, sive Imago, et Allegoria ;

dans la Conclusion, également un certain nombre de Métaphores.

Conclusione non nullae itidem Metaphorae. 


\section{NOTES}

1. Historiarum sui temporis, livre CXXV (livre non publié du vivant de de Thou ; la première édition complète de l'Historiarum est Londres, 1733). Nous reprenons le texte latin que donne Vechner (Suada Gallicana, p. 109-112), et, en modernisant les graphies, la première traduction française complète de de Thou, par l'abbé Desfontaines et al., Londres, s. n., 1734, t. XIII, p. 555-557 (la trad. Du Ryer de 1659 s'arrête au livre LVII). Cette traduction 1734 a quatre alinéas : nous repérons les trois derniers avec le signe $\$$. - Pour l'analyse de ce discours, voir dans ce numéro l'article de Giuliano Ferretti.

2. Titre latin complet: " ANALYSIS LOGICO-RHETORICA II. Orationis Gulielmi Varii ad MARIAM MEDICAEAM, novam Galliae Reginam ».

3. L'adjectif est formé comme congratulation : sun et kharis (« la grâce »), cum et gratia. Sugkhairein est du grec classique, "se réjouir, rendre grâces, féliciter»; mais sont tardifs le verbe sugkharizomai (Plutarque) et l'adjectif sugkharètikos (Philon), tout comme eukharistikos (saint Basile). Le latin ecclésiastique a eucharisticus, et Stace adresse à l'empereur Domitien un poème de remerciement intitulé eucharisticon. Que Vechner songe ou non à l'eucharistie chrétienne (eu et kharis, littéralement « la bonne grâce »), on peut faire l'hypothèse qu'avec ce recours au mot grec il dresse une grande opposition entre discours de guerre et de paix, d'où se déduit une sorte de méditation sur l'Histoire, sur l'enchaînement des causes et des effets.

4. Ou : «de félicitations », puisqu'il s'agit entre autres de louer le bonheur ou felicitas. Mais nous gardons congratulation, plus nettement relié à l'idée si importante de grâce : gratitude des sujets du royaume, bonne grâce de la nouvelle reine à souhaiter leur bonheur en retour.

5. En caractères grecs.

6. C'est-à-dire « les Parlementaires ». Vechner a bien présent à l'esprit que les parlementaires français d'Ancien Régime aiment à se comparer aux sénateurs romains. Mais Du Vair dans son discours en français (voir l'Annexe de l'article de Giuliano Ferretti) n'a aucune allusion de ce genre, qui a peu de chances d'être goûtée d'Henri IV et de lui-même: la comparaison du Parlement avec le Sénat de Rome reviendrait en effet à empiéter sur la souveraineté du monarque.

7. C'est-à-dire : une double finalité.

8. Exceptio: le substantif en latin classique n'a pas le sens de "réception "; mais c'est ici un déverbal du verbe excipio, qui a bien, lui, le sens d'" accueillir, recevoir " (Vechner ne cesse de transformer en substantifs les verbes de de Thou). — Il va de soi que les «causes finales» du $\beta$ ont plus de dignité que la cause «impulsiva» du $\alpha$, laquelle pourrait être suspectée d'être une pure «pulsion » de curiosité. L'honneur comme instrument : Malherbe dit comme "aiguillon » (latin stimulus) dans son Ode de bienvenue à la reine (v. 131-134) : «Si l'espoir qu'aux bouches des hommes / Nos beaux faits seront récités, / Est l'aiguillon par qui nous sommes / Dans les hasards précipités ».

9. Summa, le «sommaire » : c'est aussi le sommaire de l'argumentation d'ensemble du discours, énoncée ici en condensé.

10. Le siècle présent verra la longue vie de la reine, mais par définition ne pourra voir les rois issus de sa descendance, donc être la « cause efficiente » de la célébration de ces rois. Praedicatio est, comme d'habitude chez Vechner, le déverbal du praedicent sous la plume de de Thou (praedicare, " célébrer, vanter »).

11. Propriam et diuturnam : cf. Cicéron, Pomp. 48, dans un même contexte d'éloge, (optare) «ut illi proprium ac perpetuum sit ».

12. Connotatur: voir la note 25 du discours précédent. L'implicite est dans la formule «avec la grâce du Seigneur » : indication discrète mais ferme. La grâce divine est une « cause » ou " grâce 
efficiente » (cf. les formules du type " grâce suffisante » au sens de " qui suffit », etc.), elle produit un résultat effectif, elle est réalisatrice. Elle est de plus, presque par définition, la cause "principale», alors que les naissances ne sont que la cause instrumentale, l'instrument du bonheur. Voir Arnauld et Nicole (Logique, op cit., p. 301) : «Un ouvrier est la cause principale de son ouvrage, ses instruments n'en sont que la cause instrumentale », c'est-à-dire : l'ouvrier est le réalisateur véritable, la "cause première ». Bossuet, lui, assimile cause "principale » et cause «première" (op.cit., p. 442) : «Troisièmement (et c'est la plus importante de ces [sous-]divisions), on divise la cause efficiente en cause première et cause seconde. » Or, Dieu est « la seule première cause efficiente de toutes choses ", parce qu'il est «l'être même ». Le "fond de l'être » étant donné par Dieu, toutes les naissances naturelles en découlent. De ce point de vue théologique, la mère à proprement parler ne donne pas la vie (l'être) mais, comme on disait, la transmet : elle ne crée pas la vie. À leur tour, les héritiers mâles qu'elle aura créeront le bonheur solide du royaume : mais, de remontée en remontée, en dernière analyse c'est Dieu qui aura créé ce bonheur.

13. Ab opposito : sans doute un autre mot pour dire une comparatio, un parallèle terme à terme. Ici, entre notre malheur actuel (ou redouté) et le bonheur à venir dont votre arrivée donne l'espoir. Le malheur est aussi l'objection, et le bonheur, la réponse à l'objection. Le passage à la réponse est marqué par l'alinéa de la trad. 1734 : « Nous allions succomber » à notre angoisse, quand votre arrivée l'a dissipée, en y répondant terme à terme. Ce "passage par la réponse à une objection » (per) est d'autant plus nécessaire que le malheur d'abord décrit n'est pas un malheur effectif, mais seulement l'angoisse de voir finir le bonheur présent : le sentiment de sa fragilité. Un simple parallèle serait ici pour le moins bancal.

14. Hypophora se trouve en latin tardif (Martianus Capella, De nuptiis..., V, 563). En grec, c'est du vocabulaire d'Hermogène (L'invention, éd. Rabe, p. 134-130 ; L'Art rhétorique, trad. fr. M. Patillon, s. l., L'Âge d'homme, 1997, p. 244-249) : "L'hypophore est le propos [logos] de l'adversaire", l'énoncé de l'objection. Par exemple, Démosthène propose aux Athéniens de se défendre de Philippe en creusant un fossé à travers l'isthme, ce qui servirait d'immenses douves à Athènes : «Mais peut-être mes adversaires tiendront-ils encore ce genre de propos : "qu'il est difficile de creuser un fossé en travers de la Chersonèse" ». Il est difficile... est l'hypophore, suivie de la réponse à l'objection (mais non, c'est faisable). Ici, l'hypophore est : « le bonheur actuel va cesser, il n'est pas immortel». Le verbe grec hupopherein veut dire «faire chanceler, faire tomber, décliner» et au sens moral "décourager». Comme image militaire, c'est moins celle d'un obstacle explicite que d'un travail de sape. L'objection implicite ronge par en-dessous ceux qui n'arrivent pas à se l'exprimer, elle leur sape le moral.

15. En caractères grecs. Le Bailly citant chez Plutarque une construction au datif du verbe hupopherein, nous construisons : "locus ab opposito sumptus a hupophorâ [= ablatif] Regis virtutibus [= datif, complément de hupophorâ], metu et tristitia [=ablatifs, apposés à hupophorâ et l'explicitant] ». La peur est un pathos, comme la tristesse : mais la tristesse est un ajout (stoïcien) à la liste des passions dans la Rhétorique d'Aristote.

16. Ou encore : « quand on est à la tête des autres, on doit les aimer : voilà ce qu'un peuple attend comme effet (ou résultat) d'un Dirigeant ».

17. Cohortare, cohortatio, autrement dit en grec parénèse (voir "parénétique " au tout début du discours de D'Andelot), mot encore employé dans l'exégèse biblique, pour désigner le moment où l'orateur, Paul par exemple, passe à l'exhortation morale. L'exhortation est fréquente dans les péroraisons, au point d'en être presque un marqueur, puisque selon la formule d'Érasme elle enflamme, et non pas argumente (ce qu'a fait le reste du discours). En termes modernes, là où les arguments s'adressent à l'intelligence (grec logos, latin docere), l'exhortation, elle, s'adresse à la volonté pour la faire bouger (grec pathos, latin mouere). Marie de Médicis après le discours ou compliment de Du Vair « pria Dieu de lui faire la grâce de répondre aux désirs et aux espérances de la Nation »; elle est encore plus émue après celui du jésuite Suarès à Avignon (dans la 
trad. 1734, t. XIII, p. 559 ; nous soulignons) : «François Suarès complimenta la Reine au nom du Clergé, et lui souhaita un Dauphin avant l'année révolue. À ces mots cette Princesse hors d'ellemême, témoigna une envie égale aux désirs des peuples, et pria Dieu très instamment de lui accorder cette grâce. »

18. Cette unique parenthèse de Vechner indique peut-être une remarque hors texte, pour dire rétrospectivement : «la bonté et l'affection seront en effet la méthode de la Reine Marie ». C'est en tout cas la répartition des rôles bien connue : au roi la rigueur, à la reine la douceur (et la grâce). À un roi trop sévère ses sujets obéissent « sans joie ", c'est-à-dire sous la contrainte, sans adhésion ni consensus. La reine, elle, est chargée d'intercéder auprès du roi pour les pauvres sujets. On se rappelle la mise en scène de la clémence royale, comme celle des bourgeois de Calais : le roi peut ainsi afficher à la fois qu'il est sévère (et donc juste), mais pas tyrannique.

19. Dieu n'est donc pas seulement « cause efficiente principale ou première » (voir l'argument 2 et la note 12), donc cause éloignée. Il peut être aussi «cause efficiente instrumentale ou seconde ", donc cause prochaine, stimulant immédiat : l'appel ou vocation est ici l'intervention directe de Dieu dans l'histoire humaine, par ce qu'on appelait l'opération du Saint Esprit (le Saint Esprit étant Dieu en tant qu'il intervient sur terre).

20. Le grec organon correspond au latin instrumentum : la «cause organique » est donc le strict équivalent de la « cause instrumentale ».

21. $C f$. le résumé par Vechner du même discours (Suada, p. 109 ; le commonefaciens final redit le cohortatio de l'analyse, voir note 17) : «Une fois exposé le respect et l'obéissance des Sénateurs de Marseille envers la nouvelle Reine, Du Vair exprime à quel point les désirs et les vœux publics sont par cette arrivée même remplis et comblés, et il souhaite au royaume que cette félicité présente lui soit aussi durable que la bonté divine le permettra. Puis par l'éloge du Roi il laisse entendre l'inquiétude que suscitait son état de célibataire : inquiétude qu'il proclame désormais levée par l'arrivée de la Reine, en formulant l'espoir que d'elle naîtront au Roi de très nombreux héritiers. Outre la congratulation, faite au nom de tous, pour son heureuse arrivée en France, il prie pour que son règne soit longtemps bienheureux, en la louant elle-même comme Épouse du plus grand des Rois, éloge qu'il tire des divers dons qu'elle a reçus, dons de la fortune et dons de l'esprit. Enfin, sous forme d'une admonestation qui la rappelle à son devoir, il lui conseille et lui recommande d'aimer tendrement ses sujets.» («Exposito Senatorum Massiliensium erga novam Reginam cultu et obsequio, publica desideria ac vota in ipsius adventu consumpta et exhausta significat Varius, propriamque regno istam felicitatem et diuturnam maxime divinitius exoptat. Postea Rege laudato sollicitudinem ob caelibatum ejus innuit : quam Reginae adventu jam sublatam esse pronunciat, nascituros ex ea complures Regi haeredes sperans. Felicem praeterea in Galliam adventum communi nomine ipsi gratulatus, diuturnum felixque adprecatur imperium, eam tanquam Regum maximi Conjugem, a variis fortunae pariter et animi donis commendans. Sui quoque eandem commonefaciens officii, charitatem erga subditos ipsi suadet et commendat. »)

22. Quintilien cite la métonymie inverse, l'effet pour la cause (VIII, 6, 27). Chez Fontanier (Les figures du discours, op.cit., p. 79), la cause pour l'effet est la première des métonymies, avec six sous-catégories, exprimées comme ici (sa $3^{\text {e }}$ est « la Cause instrumentale et passive », ses italiques). Peut-être Vechner désigne-t-il par là la formule de de Thou sur «le temple de Thémis". Fontanier $\left(1^{\mathrm{e}}\right)$ disant en effet une «Cause suprême et divine: comme quand les Anciens disent, Jupiter, pour l'air ; Bacchus, pour le vin », on aurait ici Thémis pour la justice. Mais en quoi est-ce « externe » et « efficient »? Dans leur liste des causes efficientes, Arnauld et Nicole ne citent pas de cause efficiente externe ; Bossuet non plus. Externe de façon générale signifie «non attaché à la substance ".

23. Le mot désigne chez Quintilien (VIII, 6, 44 et $c f$. IX, 2, 46) la métaphore filée ou continuée : il suffit pour cela que la métaphore ne soit pas limitée à un seul mot. Deux mots font une allegoria.

24. En caractères grecs. 


\section{AUTEURS}

ABRAHAM VECHNER

$(1636-1704)$ 